\title{
J. SANTOS PEÑAS, A. MUÑOZ ALAMILLOS, P. JUEZ MARTEL, Y P. CORTINAS VÁZQUEZ, Diseño de encuestas para estudios de mercado. Técnicas de Muestreo y Análisis Multivariante, Madrid, Centro de Estudios Ramón Aceres, 2003
}

Bajo el título «Diseño de encuestas para estudios de mercado: Técnicas de Muestreo y Análisis Multivariante» se presenta un libro centrado en dos aspectos esenciales de la investigación con encuestas; la selección de la población a entrevistar (muestreo) y cómo extraer de la mejor forma posible la información de los datos utilizando técnicas de análisis multivariante. La obra, de más de 700 páginas, está dividida en dos partes bien diferenciadas; por lo que analizaremos en detalle cada una.

La primera parte comienza presentando -a modo de contextualización o repaso teórico- diversos conceptos estadísticos necesarios para la investigación económico-social; concretamente conceptos elementales de estadística descriptiva, de teoría de probabilidad, y de inferencia estadística.

En el segundo capítulo se realiza una presentación pormenorizada de las etapas a considerar en las investigaciones realizadas con encuestas, llevando a cabo una detallada exposición de las diversas etapas, las tareas a realizar en cada una, y las implicaciones que presenta cada etapa para las etapas posteriores. Se trata -a mi modo de ver- de uno de los mejores capítulos del libro por la exhaustividad con la que son expuestas las tareas a llevar a cabo en las distintas etapas de la investigación. Así, por ejemplo, en la «etapa previa o de planteamiento del problema» los autores desmenuzan pormenorizamente las distintas actividades a llevar a cabo (definición de objetivos, análisis y diagnóstico de la información estudiada en la fase anterior,...), al tiempo que insisten en que se trata de uno de los momentos más importantes de la investigación mediante encuestas ${ }^{1}$. Esta misma exhaustividad caracteriza el resto de apartados de este capítulo: en el segundo se muestra cómo llevar a cabo la preparación de los trabajos de campo; mientras que en el cuarto se presentan diversos procedimientos para operar con los datos e información obtenida. La «preparación de los trabajos de campo» es presentada con un nivel de profundidad que supera notablemente a la

${ }^{1}$ El autor de estas líneas, con bastantes años de experiencia en la investigación social y comercial considera que ésta es la etapa fundamental de la investigación, el momento decisivo en el que se decide lo que será la investigación. Esta idea es compartida por la 
mostrada por muchos textos específicos de muestreo ${ }^{2}$, y algo similar sucede en la «etapa de tratamiento de la información recabada en el campo". Numerosos textos de análisis de datos «parecen olvidar» las necesarias depuraciones y verificaciones que deben hacerse cuando los datos son recogidos, algo que Santos Peña y su equipo lo llevan a la perfección ${ }^{3}$. Este capítulo finaliza con un apartado dedicado al control de calidad de la investigación, en un momento en que la búsqueda de la calidad está alcanzando numerosos ámbitos de la sociedad actual (Biemer y Lyberg, 2003).

Los siguientes capítulos están dedicados a distintos tipos de muestreo utilizados profusamente en estudios de mercado: el tercero se centra en el muestreo aleatorio simple, el cuarto en el estratificado, y el quinto al muestreo por conglomerados. A continuación un capítulo donde, bajo el título «diseños muestrales prácticos", se presentan siete ejemplos de muestras reales elaboradas de principio a fin: Encuesta de Población Activa, Índice de Precios al Consumo, encuesta económica a empresas, encuesta a los consumidores para obtener información sobre la percepción

mayor parte de la literatura sobre la materia: así Díez Medrano señala, en la presentación del último libro de Kish publicado en nuestro país, que «...hacer buena investigación empírica no es cuestión de medios, sino de saber formular adecuadamente la pregunta sociológica y a partir de ahí diseñar la investigación más adecuada para alcanzar ese fin. A mis alumnos siempre les digo que es en la pregunta de investigación donde uno necesita utilizar más tiempo..." (Díez Medrano, 1995: IX). Una opinión similar, relativa a la investigación cualitativa, expresa Janesick: «siempre me sorprenden los estudiantes de doctorado y los colegas que manifiestan su intención de hacer un estudio cualitativo sin un interrogante en mente... No están preparados para diseñar proyectos cualitativos, porque no tienen ningún interrogante a partir del cual elegir los métodos apropiados» (Janesick, 1994: 210). Tama- que tienen de un producto, encuesta sobre la medición de un servicio de transporte, y una última encuesta para determinar el número de turistas de una ciudad y el gasto realizado. En suma, un estupendo capítulo que concluye a la perfección la primera parte de la obra, al sintetizar y resumir las aportaciones realizadas en los cuatro primeros capítulos, y que presenta al lector varios ejemplos reales donde se muestra toda la teoría explicada hasta el momento.

La segunda parte, dedicada al análisis multivariante de datos de encuesta (es decir, de los datos recogidos en la primera parte), comienza con dos capítulos introductorios. En el primero (capítulo 7) se explican los fundamentos básicos del programa estadístico SPSS (versiones 7, 9, 10 y 11), y es un capítulo esencial en la medida que los ejemplos utilizados en la exposición de las distintas técnicas de análisis se resuelven utilizando este programa estadístico. En el siguiente capítulo (que ocupa el octavo lugar dentro de toda la obra) se realiza una somera explicación del análisis multivariante: definición, etapas a seguir en la aplicación del análisis multivariante, condiciones que deben

yo considera que el $80 \%$ de las investigaciones que fracasan tienen sus causas en una inexacta delimitación del tema del estudio, es decir, «por ambición del tema« (1987: 50).

${ }^{2}$ El problema no es tanto que los libros sobre muestreo apenas dediquen espacio a la planificación del trabajo de campo, sino más bien que en nuestro idioma tan sólo hay publicados dos trabajos sobre esta temática (Manzano y otros 1996 y Díaz de Rada 2001a), y en un país en el que -según la asociación Española de Estudios de Mercado y Opinión (AEDEMO)- en el año 2001 se llevaron a cabo más de nueve millones de encuestas (Alos, 2002: 77). Esta situación nos obliga a considerar «¿dónde aprenden?», »¿cómo se han formado? « los encuestadores que realizaron tales encuestas.

${ }^{3}$ De hecho, hace más de diez años Villán y Bravo apuntaban que esta tendencia de «olvi- 
cumplir los datos, aspectos a tener en cuenta a la hora de elegir una determinada técnica, al tiempo que se muestran diversas técnicas de análisis multivariantes.

A partir de este momento el resto de capítulos se dedican a la explicación de una determinada técnica: así la Regresión Lineal se aborda en el capítulo 9, las Tablas de Contingencia en el 10, la Regresión Logística en el 11, los Modelos Probit en el 12, el Análisis de Varianza en el 13, el Análisis Factorial en el 14, los Test no Paramétricos en el 15, el Análisis de Conglomerados en el 16, el Análisis de Correspondencias en el 17, el Escalamiento Multidimensional en el $18 \mathrm{y}$, por último, el Análisis Conjunto en el 19. En la medida que cada capítulo está dedicado a una técnica concreta, y la estructura de todos ellos es similar, hablar de la organización de los capítulos implica presentar la estructura expositiva de la segunda parte obra. Por ello no creemos necesario realizar un análisis pormenorizado de cada capítulo en la medida que la organización de todos los capítulos es muy similar: introducción, breve explicación de los fundamentos de la técnica, ejemplos, y ejemplos resueltos con el programa estadístico SPSS.

Considerando que una recensión de un libro debe centrarse en los aspectos positivos y negativos, y no ser una mera enumeración de las partes del mismo, procedemos con la valoración de la obra. Ya hemos resaltado más arriba la excelente presentación del proceso de investigación, y el nivel de detalle con el que se explican algunas etapas como «el planteamiento del problema», "preparación de los trabajos de campo», y «etapa de tratamiento de la

do de la depuracion «, consecuencia de agilizar los procesos de producción y publicación de resultados, la rutina en las tareas diarias y el abuso de los ordenadores, está generando un descuido en los trabajos de depuración y limpieza, olvidando que estos pueden tener un información recabada en el campo». Respecto a la segunda parte, me parece magnífica la exposición que los autores realizan de las distintas técnicas, y la forma en la que resumen toda la explicación en los ejemplos resueltos con el SPSS. Mi experiencia docente en la asignatura Técnicas Avanzadas de Investigación Social, unida a la elaboración de un libro sobre la materia (Díaz de Rada, 2002), me ha llevado a analizar muchos libros sobre análisis multivariante, $\mathrm{y}$ he de decir que en éste los autores resuelven perfectamente las complicadas explicaciones que precisan determinadas técnicas. A estos aspectos a los que hay que añadir la «valentía» de la editorial de publicar un volumen de 708 páginas, con los problemas para comercializar estos trabajos tan voluminosos.

Por otro lado, y aunque la obra termina con una serie de referencias bibliográficas, consideramos que se echa de menos un mayor número de referencias que permitan al lector interesado llevar a cabo una ampliación de conocimientos sobre cada uno de los temas tratados. Este hecho adquiere más importancia cuando se considera el público objetivo de este libro: lectores con escasos conocimientos en la materia ( $\mathrm{y}$ que en numerosas ocasiones necesitan orientación sobre las obras a consultar para ampliar conocimientos ${ }^{4}$ ) y, más concretamente, alumnos del curso de postgrado Diseño y Tratamiento estadístico de Encuestas para Estudios de Mercado y Sondeos de Opinión 5 .

Quizás algún lector puede considerar que falta un espacio destinado a la elaboración de cuestionarios y otros instrumentos para la recogida de información, pues-

importante impacto en la calidad de los datos recogidos (Villán y Bravo, 1990: 15).

${ }^{4}$ No debemos olvidar que muchos de los libros sobre estas temáticas precisas de un elevado conocimiento estadístico-matemático.

5 http://www.uned es/experto-tratamientoestadistico-encuestas/ 
to que la primera parte-dedicada a la recogida de información- se centra fundamentalmente en el proceso de recogida: preparación trabajos de campo, trabajo de campo, tratamiento de la información recabada en el campo. No estamos de acuerdo con esa percepción puesto que, desde nuestro punto de vista, el enorme espacio necesario para la explicación del cuestionario supondría un pérdida de perspectiva del proceso de investigación. Por otro lado, dedicar poco espacio a la elaboración de cuestionarios supondría tratar el tema sin la necesaria profundidad.

Una estrategia similar fue utilizada por el autor de estas líneas en Técnicas de Análisis de Datos para Investigadores Sociales. En aquel momento consideramos que una adecuada exposición del proceso de investigación suponía «hacer concesiones» y no explicar en detalle cada una de sus etapas, en la medida que las exposiciones pormenorizadas implican pérdidas de fluidez en la explicación de un proceso. Los comentarios de los lectores han constatado la adecuación de esta estrategia, si bien algunos de ellos sugirieron la conveniencia de incluir unas notas sobre la elaboración del cuestionario en alguna edición posterior de la obra. Fruto de esta demanda nace Diseño y Elaboración de Cuestionarios para la Investigación Comercial. Las 174 páginas de este trabajo nos llevan a constatar que haber incluido unas notas sobre la elaboración del cuestionario hubiera supuesto proporcionar una información muy parcial del tema, con la consecuente pérdida de fluidez en la explicación del proceso de investigación.

\section{REFERENCIAS BIBLIOGRÁFICAS.}

ALÓS, J. (2002). «Industria de los estudios de mercado en España 2001», Investigación y Marketing, vol. 76, pp. 75-78.

BIEMER, P.P. y LYBERG, L.E. (2003). Introduction to Survey Quality. Nueva York: Wiley.

DÍAZ DE RADA, V. (1999). Técnicas de Análisis de Datos para Investigadores Sociales. Madrid: Rama.

DÍAZ DE RADA, V. (2001). Organización y gestión de los trabajos de campo con encuestas personales y telefónicas. Barcelona: Ariel.

DIAZ DE RADA, V. (2001). Diseño y elaboración de cuestionarios para la investigación comercial. Madrid: Esic.

DIAZ DE RADA, V. (2002). Técnicas de Análisis Multivariante para investigación social y comercial. Madrid: Rama.

DÍEZ MEDRANO, J. (1995). «Prólogo a la edición española», en L. KISH, Diseño estadístico para la investigación. Madrid: Centro de Investigaciones sociológicas, monografía número 146, pp. IX-X.

JANESICK, V.J. (1994), «The dance of qualitative research design», en $\mathrm{N}$. DENZIN y Y. LINCOLN (Eds.), Handbook of Qualitative Research. Thousand Oaks, California: Sage, pp. 209-219.

MANZANO ARRONDO, V.; ROJAS, A.J. y FERNÁNDEZ, J.S. (1996). Manual para encuestadores. Barcelona: Ariel.

TAMAYO, M. (1987). El proceso de investigación científica. México: Limusa.

VILLAN CRAIDO, I. y BRAVO CABRIA, M.S. (1990). Procedimiento de Depuración de Datos Estadísticos, Seminario Internacional de Estadística en Euskadi, EUSTAT, Vitoria 1990.

Vidal Díaz de Rada 